\title{
Optimization of a novel micro-opto-X-ray imaging lens
}

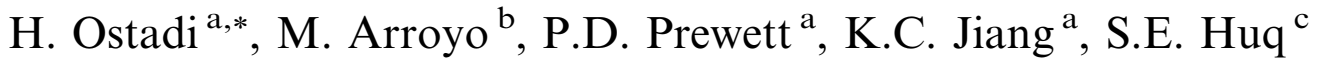 \\ ${ }^{a}$ Department of Mechanical Engineering, University of Birmingham, Birmingham B15 2TT, UK \\ ${ }^{\mathrm{b}}$ Department of Applied Mathematics III, Catalunya Polytechnic University, Barcelona, Spain \\ ${ }^{\mathrm{c}}$ STFC Rutherford Appleton Laboratory, Chilton, Didcot OX11 0QZ, UK
}

Received 8 October 2007; received in revised form 17 January 2008; accepted 21 January 2008

Available online 9 February 2008

\begin{abstract}
A mechanically deformable reflection transmission MOEMS system is capable of focusing X rays to sub micron spots. This paper considers the geometry of the proposed deformed slotted microcantilever lens element under thermally derived strain, using finite element analysis. The work shows that an optimized MOEMS design using a slotted polyimide/gold thermal bimorph cantilever is capable of achieving ideal geometry with a larger than expected number of focusing slots up to 111 at $2 \mu \mathrm{m}$ width.
\end{abstract}

Keywords: Micro opto electro mechanical systems; X ray focusing; Microcantilever; Thermal bimorph; Finite element analysis

\section{Introduction}

Micro-opto-electro-mechanical systems (MOEMS) are of interest for biomedical applications. For instance, studies of the Bystander effect require controlled irradiation of biological cells with focused X-rays to reveal the mechanisms occurring $[1,2]$.

A MOEMS system, designed for this application, is an integration of a flexible microlens, sensor and mechanical actuation mechanism with an electro-mechanical control system to set the require focal length. The system has been given the acronym MOXI, standing for micro-opto-X-ray Imaging. The design and microfabrication of a one dimensional (1-D MOXI) focusing MOEMS system with micro slots for use in X-ray analysis of biological cells was reported in [3]. A 2-D MOXI adaptive optics system for $\mathrm{X}$-ray focusing was suggested in [4]. The present paper focuses on details of the geometry of the deformed slotted micro-beam lens element under thermally derived strain, using finite element analysis, and suggests an optimized MOEMS design, giving prescribed curvature of the lens

\footnotetext{
* Corresponding author.

E mail address: ostadi@gmail.com (H. Ostadi).
}

through changing the number and the dimensions of the slots.

A 1-D reflective focusing element forming part of a MOXI/MOEMS providing focusing in one direction only is shown in Fig. 1. This is intended for use with a second identical element in cross-coupled Kirkpatrick Baez mode to produce a sub micron spot focus [5].

\section{Modeling}

The bimorph microlens is a sandwich of layers of polyimide and gold with an embedded metal micro-heater. The difference in linear thermal expansion between the two materials produces a displacement perpendicular to the strain plane. An analytical model assuming cylindrical curvature is inadequate to describe experiments and FEA had to be used. The micro slotted cantilever thermal analysis showed that the temperature propagation is very fast micro seconds and in a controlled area such as a clean room, a homogeneous temperature over the cantilever in the heating and cooling process could be assumed [6]. The chosen software was the SOLID 45 3-D element, which provides for eight nodes with three degrees of dimensional freedom at each node, viz. translations in the $x, y$, 


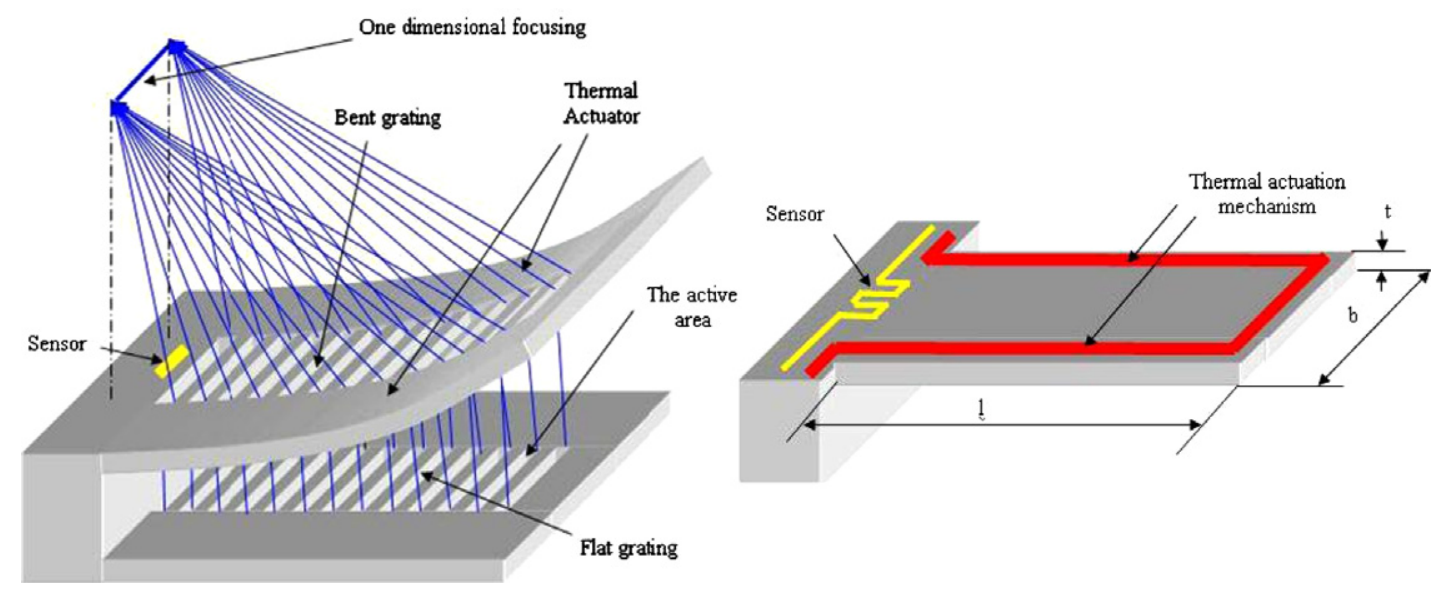

Fig. 1. Focusing action of $1 \mathrm{D}$ MOXI/MOEMS reflection transmission lens

and $z$ Cartesian directions in the associated ANSYS programme. The dependent variables include creep, swelling, stress, deflection, and strain [7]. In this study, both linear and non-linear analysis were employed and compared with the experimental results from [3]. The model beam consists of polyimide of thickness $7 \mu \mathrm{m}$ and gold of thickness $1 \mu \mathrm{m}$.
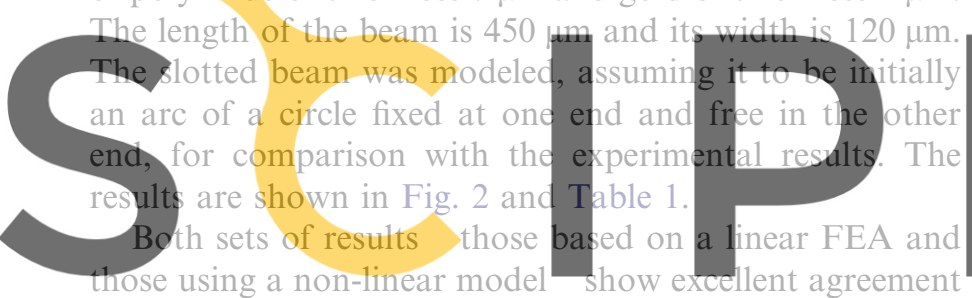

with experiment at temperatures up to $90^{\circ} \mathrm{C}$. However, at

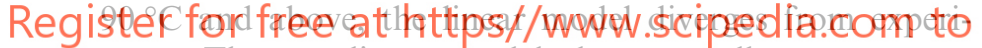

ment. The non-linear model shows excellent agreement with experiment up to and including a temperature of $100{ }^{\circ} \mathrm{C}$, but this model too shows strong divergence from theory at $100{ }^{\circ} \mathrm{C}$. This is consistent with the observations

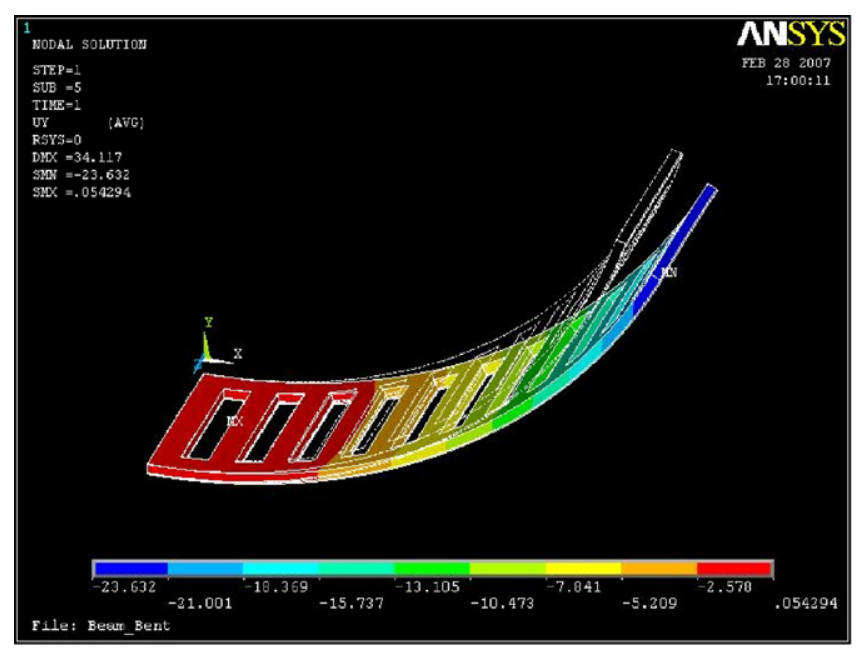

Fig. 2. FEA results showing microcantilever deformation vs. temperature. The microcantilever dimensions are $450 \times 120 \times 7 \mu \mathrm{m}$; the skeleton shape is the undeformed prestressed bimorph after fabrication with 11 slots.

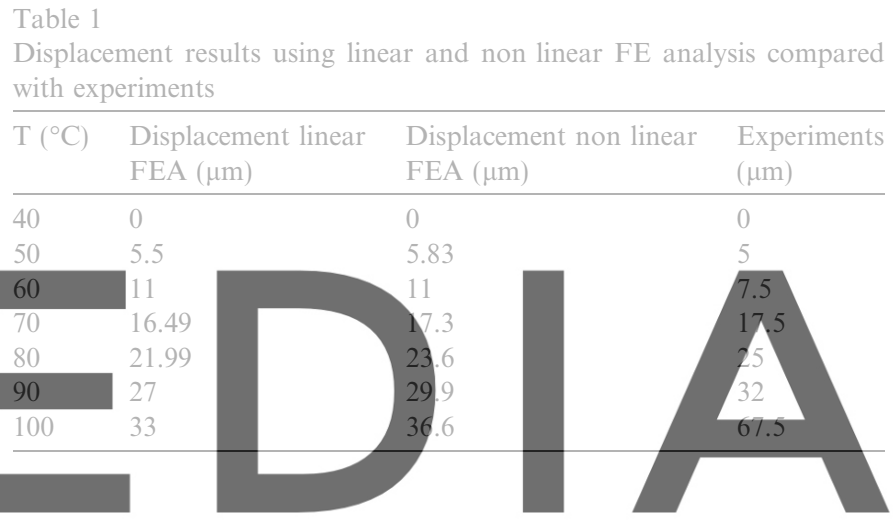

of Al Aioubi et al. [8], who report irreversible distortions

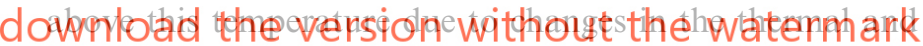
mechanical properties of the polyimide. The thermal expansion coefficient of polyimide increases rapidly about $40 \%$ above $100{ }^{\circ} \mathrm{C}[9]$.

\subsection{Optimization}

Optimization of the microlens, from the standpoint of its mechanical properties, is an important issue which can be resolved by FEA. The key question is how does the stress in the microcantilever vary with the number of slots? The number of slots should be the maximum possible in order to maximize X-ray transmission, but this is limited by the need to maintain structural integrity under thermal actuation. The modeled dimensions are $450 \mu \mathrm{m}$ length, $250 \mu \mathrm{m}$ initial displacement, thickness $8 \mu \mathrm{m}$. In this optimization process the slot length is fixed; only the number of slots and their width are varied. The proven non-linear FEA approach was used. The data from finite element analysis of slotted beams with 1, 3, 5, 7 and 11 slots are given in Table 2.

The finite element analyses of the slotted beams are compared with a perfect circle. In this analysis the widths of slots are $20 \mu \mathrm{m}$ and the length $90 \mu \mathrm{m}$. Another assumption in this section is that, the distance between the slots is equal to the distance of the both end slots to the both free 
Table 2

Curvature data obtained from finite element analysis of slotted beams with $1,3,5,7 \& 11$ slots, compared with perfect circles

\begin{tabular}{ll}
\hline Maximum deviation from perfect circle $(\mu)$ & Number of slots \\
\hline 1.66 & 11 \\
2.2 & 7 \\
2.4 & 5 \\
2.6 & 3 \\
2.7 & 1 \\
\hline
\end{tabular}

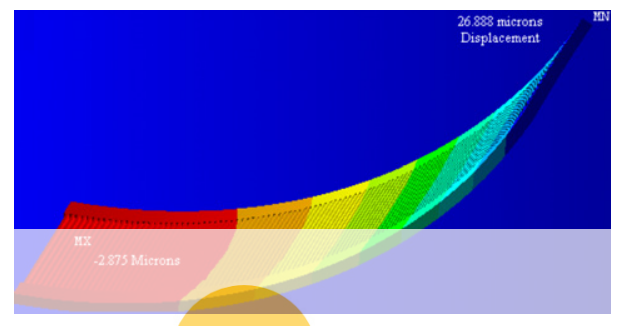

Fig. 3a. FE analysis for an optimized microlens of length $450 \mu \mathrm{m}, 111$ slots, $2 \mu \mathrm{m}$ wide. Minimum displacement is $-2.875 \mu \mathrm{m}$ and the maximum is $26.888 \mu \mathrm{m}$.

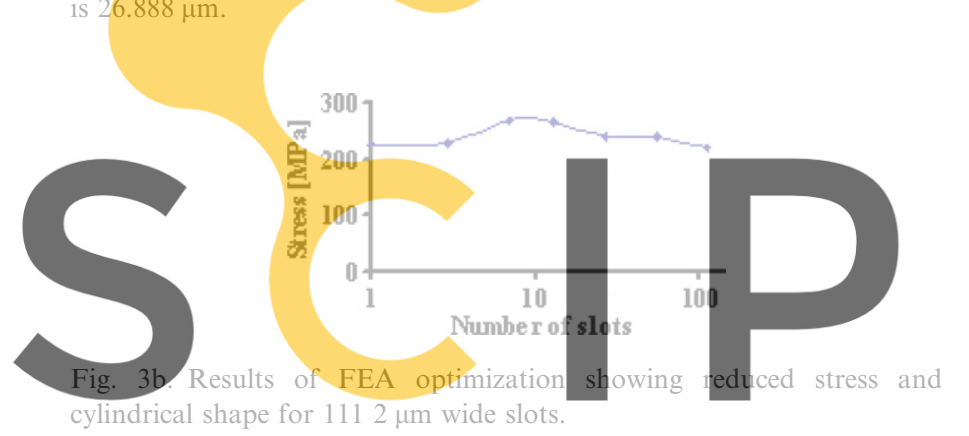

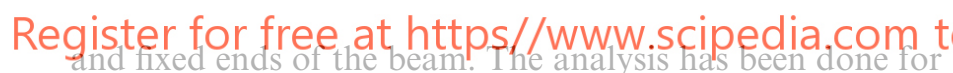
several samples as below, all for the same temperature rise, $\Delta T \quad 45^{\circ} \mathrm{C}$.

- 1, 3, 5, 7, 11 slots, $20 \mu \mathrm{m}$ wide.

- 1, 3, 7, 13, 27 slots, $8 \mu \mathrm{m}$ wide.

- $1,3,7,13,27,55$ slots, $4 \mu \mathrm{m}$ wide.

- $1,3,7,13,27,55,111$ slots, $2 \mu \mathrm{m}$ wide.

From the FEA, there is a clear number of slots for each slot width at which the internal stress in the structure is maximized. Also the curvature, which is arguably the most important parameter in the optimization of the microlens is closer to a perfect circle as the number of slots increases.
Since microfabrication using optical lithography restricts the readily achievable slot width to approximately $2 \mu \mathrm{m}$ and bearing in mind the foregoing, an optimized structure for our $450 \mu \mathrm{m}$ long microcantilever would have 111 slots of width $2 \mu \mathrm{m}$. The results of non-linear FEA analysis for such a structure are shown in Fig. 3a. The stresses are not strongly dependent on the width of slots, depending rather on their number (Fig. 3b).

\section{Conclusions}

A series of simulations using FEA analysis has shown the dependence of tip deflection, form and internal stresses on structure and temperature for microcantilever bimorph structures as designed for an X-ray focusing MOEMS (1-D MOXI). The critical importance of the number of slots in obtaining the spherical form necessary for aberration free spot formation has been shown. The superiority of non-linear FEA compared with linear FEA has been revealed by the study, but a simple analytical model is surprisingly good. An optimized structure containing 111 slots $2 \mu \mathrm{m}$ wide is shown to give a thermally actuated form very close to a perfect circle, as required for the 1-D MOXI MOEMS
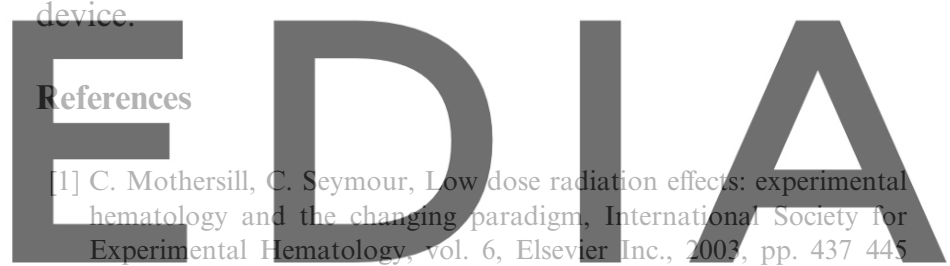
(June 31)

[2] K. Atkinson, Methods of efficiently focusing TiK $\alpha$ X Ray in low

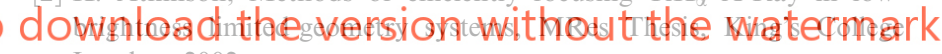
London, 2002.

[3] M.Y. Al Aioubi, P.D. Prewett, S.E. Huq, V. Djakov, A.G. Michette, Microclectronic Engincering 84 (2007) 12521255.

[4] M.Y. Al Aioubi, P.D. Prewett, S.E. Huq, V. Djakov, A.G. Michette, Microelectronic Engineering 83 (2006) 13211325.

[5] P. Kirkpatrick, A.V. Baez, Journal of the Optical Society of America 38 (1948) 766.

[6] H. Ostadi, M. Arroyo, P.D. Prewett, S.E. Huq, Finite element study of a micro opto $\mathrm{X}$ ray imaging lens for biomedical applications, in: 2007 ASME International Mechanical Engineering Congress and Exposi tion, IMECE2007, Washington, USA.

[7] ANSYS Release 9 Documentation, SOLID70 and SOLID45 Elements Description.

[8] M.Y. Al Aioubi, V. Djakov, S.E. Huq, P.D. Prewett, Microelectronic Engineering 7374 (2004) 898903.

[9] <http://www.matweb.com>. 